\title{
ACTUALIZACIÓN DE LA ÓPERA Y SUS NUEVOS MODELOS DE COMUNICACIÓN DIGITAL
}

Ivan Lacasa-Mas e Isabel Villanueva-Benito

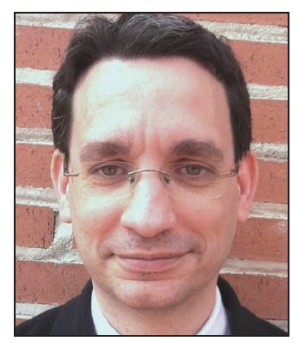

Ivan Lacasa-Mas, doctor por la Univ. Autònoma de Barcelona (UAB), es profesor de teoría y ética de la comunicación en la Fac. de Ciències de la Comunicació de la Univ. Internacional de Catalunya (UIC), de la que actualmente es decano. Ha sido profesor visitante en el Institut für Kommunikationswissenschaft und Medienforschung de la LMU-München. Investigador del grupo Labcom y del proyecto CSO200913713-C05-02 del Mo de Ciencia e Innovación, así como del proyecto competitivo Walter Hagemann (1900 bis 1964). Eine Fallstudie zum Zusammenhang zwischen Habitus, Kapital und kommunikationswissenschaftlichem Arbeiten, de la Deutsche Forschungsgemeinschaft (DFG). Investiga los nuevos medios y las nuevas audiencias, así como los procesos de mediatización de las realidades sociales.

Universitat Internacional de Catalunya - Facultat de Ciències de la Comunicació Immaculada, 22. 08017 Barcelona, España lacasa@cir.uic.ess

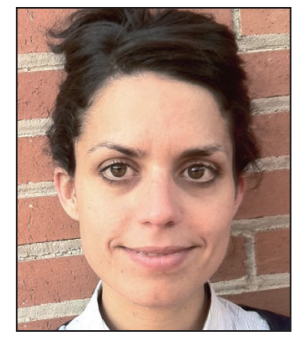

Isabel Villanueva-Benito prepara su tesis doctoral sobre ópera, cine y nuevas tecnologías. Es profesora en la Facultat de Ciències de la Comunicació de la Universitat Internacional de Catalunya (UIC). Licenciada en comunicación audiovisual y publicidad y relaciones públicas, graduada superior de canto, se ha especializado en dos líneas de investigación: la composición musical para cine y nuevos medios, y la interpretación operística. En la actualidad compagina su carrera profesional con su labor como docente universitaria. Participa en el proyecto nacional I+D "Música i imatge. Art, tecnologia i comunicació audiovisual".

Universitat Internacional de Catalunya - Facultat de Ciències de la Comunicació Immaculada, 22. 08017 Barcelona, España isabeluic@gmail.com

\section{Resumen}

La industria de la ópera quiere renovar sus audiencias y ha emprendido estrategias basadas en la digitalización y la comunicación audiovisual para conseguirlo. Explora modos de actualizar su política informativa y modernizar su imagen para atraer y fidelizar a nuevos públicos. Utiliza internet para sacar el espectáculo operístico de los escenarios y acercarlo a la vida cotidiana de los usuarios, gracias al vídeo bajo demanda y a las retransmisiones de tipo live streaming. Usa los dispositivos móviles para aumentar su presencia compartible, imita las tendencias comerciales audiovisuales con el objetivo de despertar entusiasmo y se constituye en mundo de referencia a través de la redes sociales. Ve en su actualidad y su integración en la sociedad una manera de volver a ser relevante.

\section{Palabras clave}

Ópera, Artes, Mediatización, Actualización, Comunicación, Audiovisual, Internet, Públicos, Dispositivos móviles.

Title: The update of the opera and its new models of digital communication

\begin{abstract}
The opera industry wants to renew its audience and has undertaken several strategies based on digitalization and audiovisual broadcasting to achieve it. In order to attract and create a stronger bond with new audiences, it is exploring different ways to update its information policy and modernize its image. By means of Internet, it has managed to get the opera show offstage, bringing it closer to the daily lives of its spectators thanks to video on demand (VOD) and to live streaming broadcastings. It has made the most of mobile devices to enhance their shareable presence, imitating the audiovisual commercial trends to arouse enthusiasm among its viewers and creating social networks to offer the audience a referential background. Finally, the opera industry looks upon its current state and its social integration as a means of regaining its relevance.
\end{abstract}

\section{Keywords}

Opera, Arts, Mediatization, Updating, Communication, Audiovisual, Internet, Audiences, Mobile devices.

Lacasa-Mas, Ivan; Villanueva-Benito, Isabel. "Actualización de la ópera y sus nuevos modelos de comunicación digital”. El profesional de la información, 2012, julio-agosto, v. 21, n. 4, pp. 413-418. 


\section{Introducción}

La ópera siente que tiene un problema de actualidad. A ojos de muchos representa un género musical lejano y propio de otra época (Adorno, 2006; Tambling, 1987). Pero, como todo arte, la ópera es una realidad abierta que desea tener público (Eco, 1985, pp. 30, 66-67). Para ampliar y renovar sus audiencias ha decidido digitalizar y audiovisualizar su comunicación. Parece ser consciente de que en una sociedad mediática la actualidad no es tanto una propiedad de las cosas, como del mensaje que de ellas habla; una proporción entre una información emitida y las expectativas de los receptores (Merten, 1999, p. 203). Sabe que la batalla que tiene que dar no consiste en alcanzar una mayor relevancia musical. Su reto ahora es despertar un mayor interés en el público. Entiende que sólo si recibe más atención, será considerada actual (Ruhrmann, 2005, p. 68). Por ello se sirve de los medios digitales de comunicación para integrarse en los mundos de los nuevos públicos (Silverstone, 1994, p. 3; García-Avilés, 2009). Sale de los teatros e intenta formar parte de la vida de las personas, de su archivo simbólico, ser incluida en la imagen que la sociedad construye de sí misma y de su tiempo (Hoffmann, 2011, p. 273). La ópera busca cómo adaptar su espectáculo presencial, en directo y en comunidad, a los nuevos modelos de consumo, sobre todo a los que se dan en internet, un aliado perfecto debido a su ubicuidad (Carroll; Foth; Adkins, 2010, p. 147).

La ópera explora vías para adaptar el espectáculo presencial a los nuevos modelos de consumo en internet

\section{Distribución online de ópera}

La ópera es el arte escénico narrativo de mayor duración temporal (Radigales, 1999; Parker, 1998; Alier, 2008). Aunque a priori internet no favorezca el consumo natural de un tipo tan largo de espectáculos, su virtualización sí consigue emular fielmente la presencialidad y el vivo de la obra original (Heyer, 2008, p. 601; Chavarría, 2010, pp. 40-41). Ambas características, presencialidad y directo, son consideradas notas esenciales del género musical (Parker, 1998, p. xi; Roselli, 1998, p. 482). Por fortuna la Red es capaz de recrearlas. Consciente de ese hecho, la industria operística apuesta por dos servicios en su distribución online: el vídeo bajo demanda (VOD) y las retransmisiones de tipo live streaming.

EI VOD es la primera gran herramienta de distribución online de contenidos de los teatros de ópera de prestigio internacional ${ }^{1}$. Su primacía responde a razones económicas: al ser de pago, el VOD aporta una nueva ventana de explotación para la industria. Esa dimensión comercial despierta en los organismos cierta premura por digitalizar, actualizar y preservar su propio archivo de materiales operísticos históricos para disponer de un buen catálogo.

Un claro inconveniente del VOD es que su formato carece de la capacidad de emular la experiencia del espectáculo en vivo. Todo lo demás son ventajas: implica una liberación de las ataduras de tiempo y espacio, reduce los costes de distribución, facilita el encapsulado de la obra y aumenta la presencia del producto operístico en la vida de los usuarios. Además, permite navegar con facilidad por él, elaborar listas de favoritos o elegir la calidad de la reproducción (Huang; Li; Ross, 2007; Orihuela, 2002).

La oferta de vídeos operísticos bajo demanda intenta despertar el interés de audiencias nuevas y más mediáticas. Pero, hoy por hoy, es un servicio que parece responder mejor a los usuarios del arte ya fidelizados, al consistir su punto fuerte en la capacidad de inducir nostalgia y recuerdo en aquellos públicos que asistieron al teatro, de comparar versiones o de recuperar detalles que no pudieron apreciar en la representación original (Wasserman, 2009).

El teatro pionero en ofrecer VOD fue el Metropolitan Opera House de Nueva York (Met), que en 2008 lanzó el primer servicio de pago, denominado Metplayer. El proyecto, hoy rebautizado como Met opera on demand, constituye una amplia mediateca de 300 vídeos en calidad VHS y HD, además de archivos de audio. Desde el lanzamiento de ese producto, teatros de prestigio como el Teatro Real de Madrid, mediante su Palco digital, o la Deutsche Oper Berlin, han impulsado iniciativas similares. El resto de organismos están siguiendo también, en mayor o menor medida, esta nueva línea de comunicación audiovisual. Ofrecen gratis fragmentos online de sus propias obras, logrando así difundir el es-

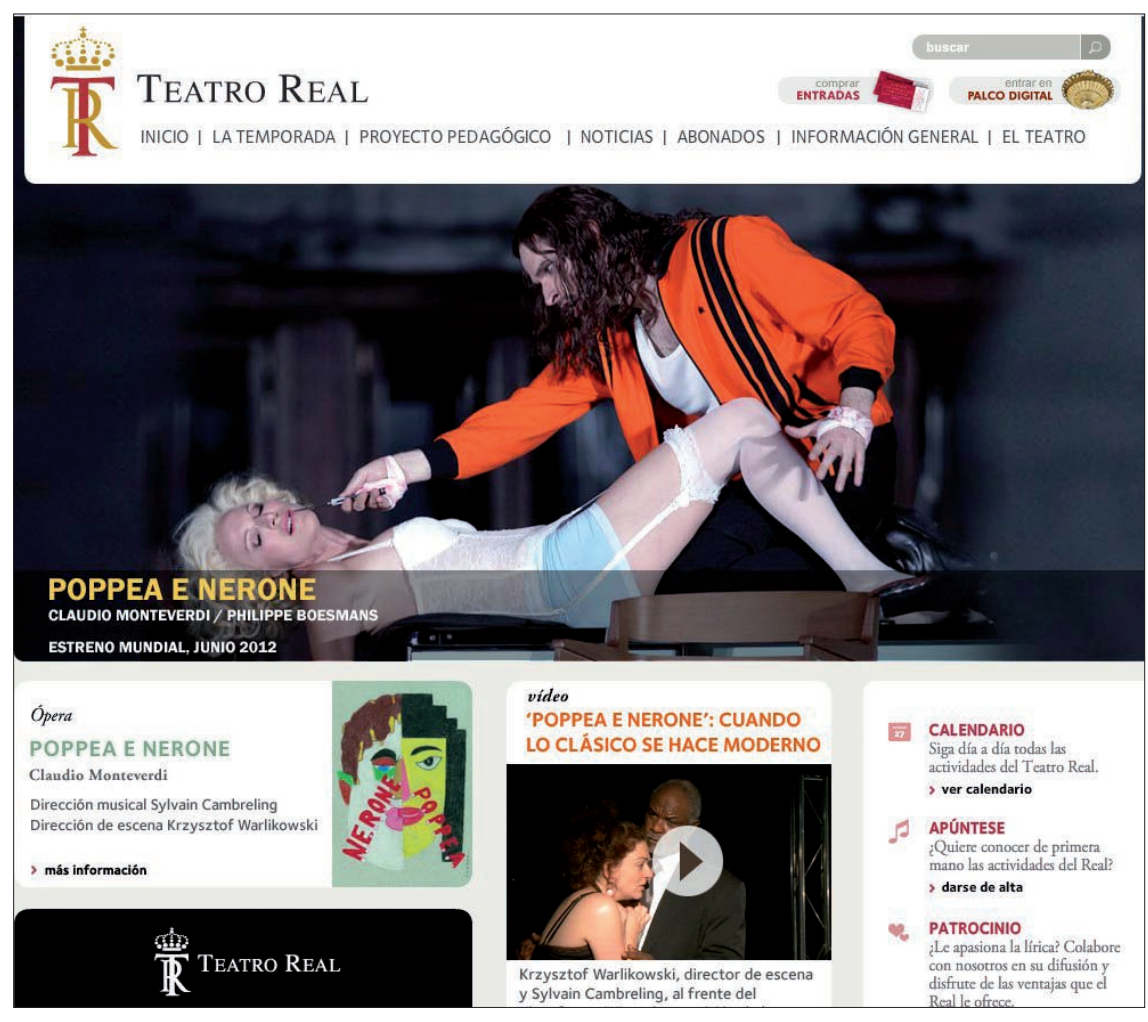

El Teatro Real de Madrid es pionero en ofrecer vídeo online bajo demanda. http://www.teatro-real.com 


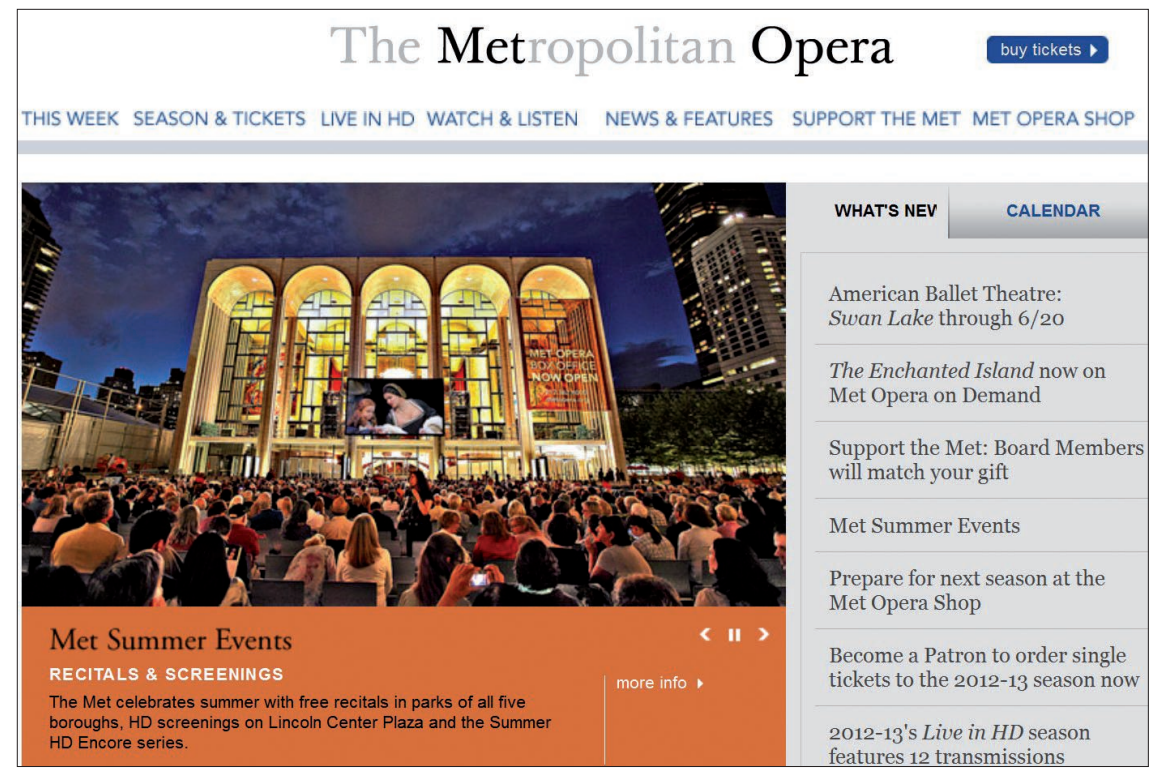

http://www.metoperafamily.org habilitada por proveedores de infraestructura como las instituciones internacionales de tecnología de banda ancha I2Cat o GARR. Logran llevar el espectáculo hasta el consumidor final y posibilitan que participen virtualmente en él (Berte; Hauttekeete; Mechant; Nulens, 2010). Como ellas mismas reconocen (Bakhshi; Pugh, 2011), si quieren seguir actualizándose, las industrias culturales deberán colaborar cada vez más estrechamente con esos terceros agentes tecnológicos, ya que, desde el punto de vista artístico, el servicio in live streaming parece el más idóneo para actualizar la ópera: transporta la dimensión vivencial del directo y provoca la copartipación en tiempo real (Heyer, 2008, p. 601). pectáculo, y promocionar el teatro y la temporada en curso (Radigales; Villanueva, 2011). Algunos optan por crear apartados audiovisuales propios. Otros prefieren ofrecer sus contenidos a través de plataformas generalistas como YouTube. Ejemplos del primer caso son la sección Opera video impulsada por la Opéra National de Paris, el servicio Play de la Sydney Opera House, o el apartado Video gallery de La Scala de Milán. Ejemplos del segundo son los canales del Chicago Opera Theater o de la Royal Opera House en YouTube, que ya cuentan con seis años de antigüedad.

El segundo gran servicio online que ofrece la ópera para difundir su arte es el live streaming de consumo doméstico, una iniciativa todavía joven, que está fuertemente condicionada por factores económicos y tecnológicos, al depender de la sincronización temporal con el espectáculo original (Serra, 2011). De ahí que, hoy por hoy, sus principales promotores sean los grandes festivales internacionales y las empresas de infraestructura tecnológica como tercer agente. En 2008 el festival de Bayreuth lanzó por primera vez esta nueva forma de atraer virtualmente al encuentro a nuevos públicos. Ofreció los estrenos en directo desde su página web. Consiguió rentabilizar al máximo su programa temporal, logró internacionalizarse y convirtió a la ópera en un hecho noticiable durante los meses en que se celebró (De-Diego, 2010, p. 4). La iniciativa fue repetida e imitada por organizaciones como The Glyndebourne Festival.

Además de los festivales líricos, están surgiendo empresas intermediarias especializadas en retransmitir el vivo, tal como hemos avanzado más arriba. Proliferan con éxito las plataformas de difusión digital y los canales de televisión online, Medici.tv o ClassicalTV son dos ejemplos. Su red de señal es

\section{"En la Red la ópera ofrece un mundo de referencia para sus nuevos públicos"}

\section{Presencia informativa online de la ópera}

La ópera no pretende actualizarse únicamente con medidas de distribución online de sus obras. Al ser un arte complejo y necesitado de cierto deseo añadido de aprender (Smith, 1993, pp. 21-61; Fülfe, 2011), ha emprendido también la creación de un contexto informativo online que sirva de referencia y facilite el entendimiento de sus obras y de su mundo a los nuevos públicos.

Para ello impulsa iniciativas comunicativas múltiples y heterogéneas, que resulta imposible recoger de manera exhaustiva en este análisis. Ilustraremos sólo las principales tendencias:

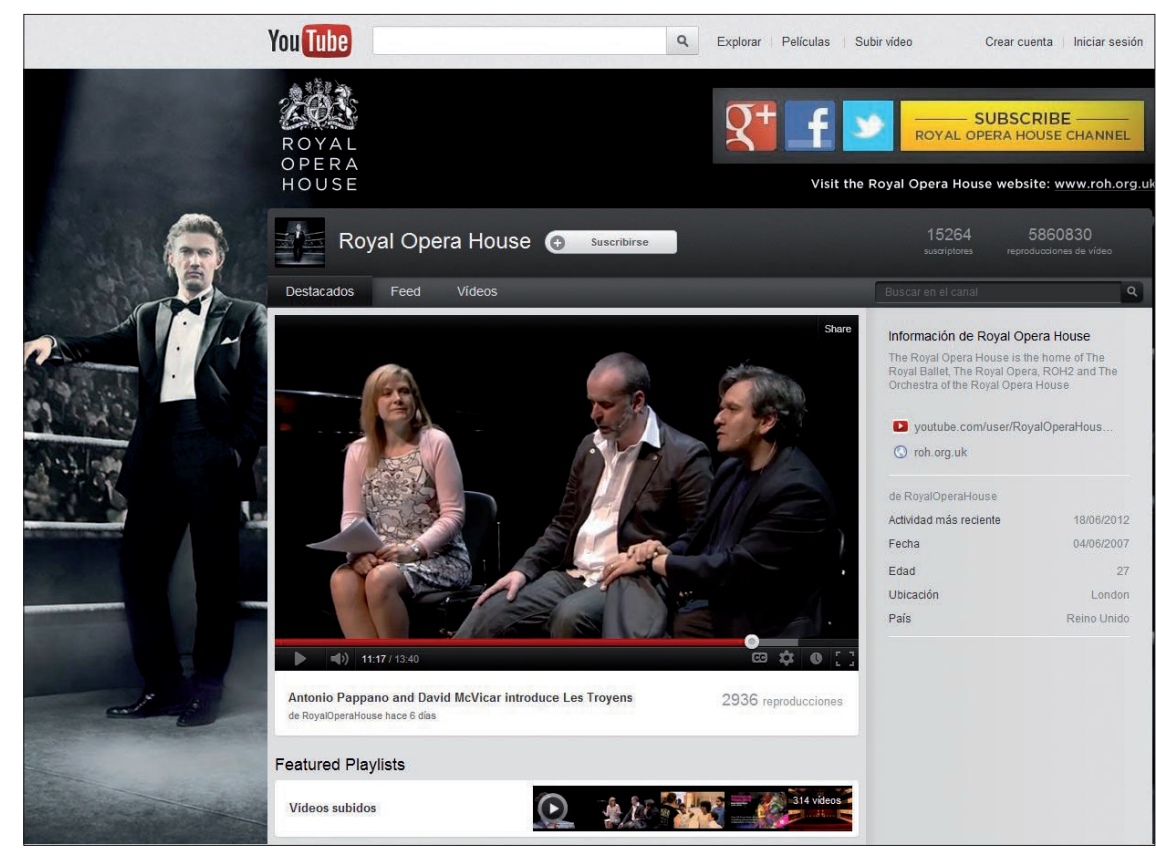

Canal de la Royal Opera House en YouTube, http://www.youtube.com/user/RoyalOperaHouse 
1) Paulatina adaptación de los sitios web de las instituciones operísticas a los nuevos dispositivos móviles. El Teatro Real, la Opéra National de Paris, el Met, o la Sydney Opera House ya han lanzado aplicaciones para iPad e iPhone. Con ellas refuerzan su presencia como referentes en el día a día de las personas y las mantienen informadas puntualmente. Aunque de momento sólo transmiten los mismos contenidos que las webs oficiales, con esta estrategia facilitan en algo la integración social del arte y permiten una mayor interactuación entre pares, gracias a las posibilidades que ofrecen de compartir vídeos o de enviar directamente enlaces por correo electrónico.

2) El número de aplicaciones para tablets aumenta cada día. Sin embargo,

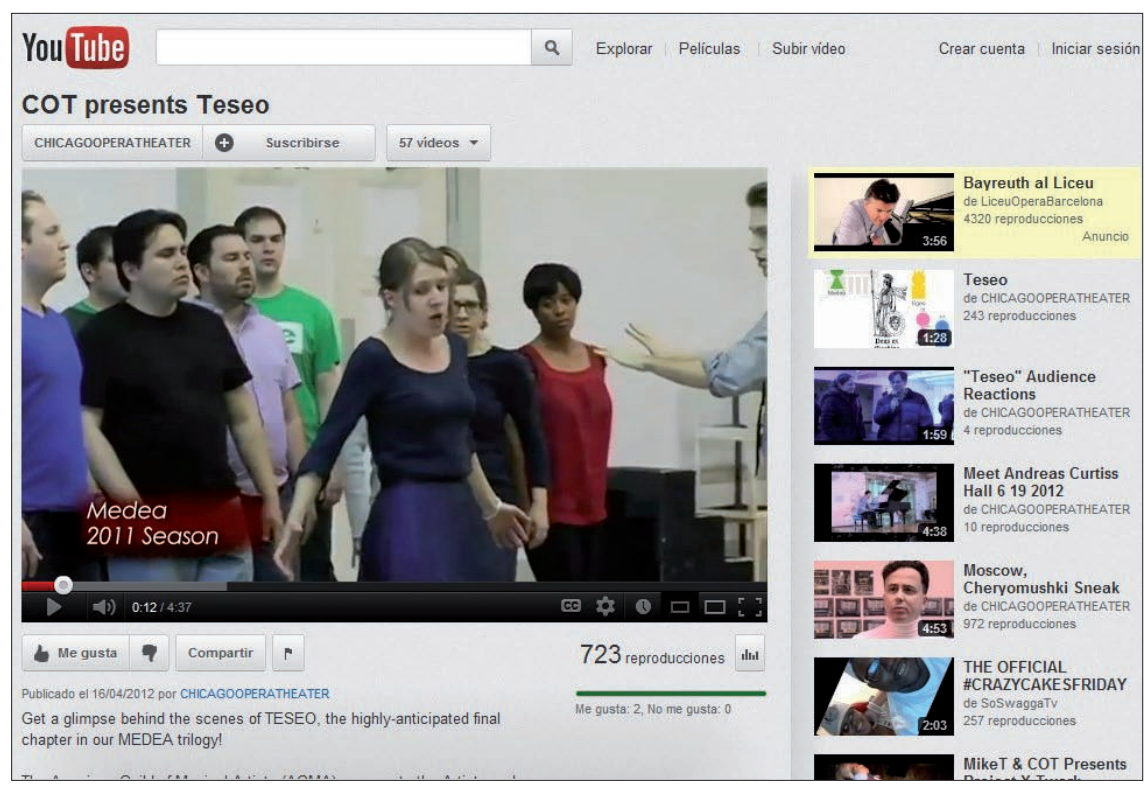

Chicago Opera Theater (COT), http://www.youtube.com/user/chicagooperatheater el desarrollo de servicios específicos desligados de la web es aún casi inexistente. Merecen destacarse dos excepciones. En 2011 se lanzó el primer juego operístico para iPhone, llamado The show must go on. Se trata de la primera aplicación lúdica patentada por un teatro oficial, la Royal Opera House de Londres. Por otro lado, en marzo de 2012 el Met presentó la primera mediateca para iPad denominada Met opera on demand. En caso de seguir confirmándose la incipiente tendencia a visionar productos audiovisuales de larga duración a través de los nuevos dispositivos portátiles, esta fórmula de mediateca móvil podría estar avanzándose al futuro (Viacom, 2012).

\section{Las redes sociales permitirán fidelizar a las nuevas comunidades en torno al es- pectáculo}

3) Para llenar de contenidos esos dispositivos, la ópera, influenciada por las estrategias del marketing de las artes, tiende a imitar los productos de consumo audiovisual más de moda (Colbert; Cuadrado, 2003; McLean, 1994; Scheff; Kotler, 1996). Imita aquellos formatos que facilitan el consumo en la Red de sectores como el cine, la televisión o la publicidad: vídeos de corta duración, estéticamente visuales y fácilmente compartibles (Muro, 2006, p. 157; Calvi; Santini, 2010, pp. 1-4).

La influencia de la televisión se observa también en la creación de reality shows ${ }^{2}$, en la aparición de reportajes sobre la vida íntima de los $\operatorname{divos}^{3}$ o en la creación de videoclips líricos. Del cine, la ópera imita el uso de trailers ${ }^{4}$ de las temporadas líricas y del material extra online -making of de los espectáculos, biografías de cantantes y compositores o declaraciones del equipo artístico- (De Diego, 2010, pp. 1-2; Sheil, 2012, pp. 143-150). De la publicidad y el marketing online incorpora tendencias novedosas de impacto en la Red, como son los mensajes virales, los spots online y la creación a bajo coste de lipdubs y flashmobs para promocionar las obras en cartel ${ }^{5}$.
Gracias a las iniciativas anteriores, el consumidor aprende el valor del arte como realidad más compleja de lo que la música o el texto del espectáculo concreto permiten apreciar. También contextualiza el sentido de la obra en un marco de actualidad (Sheil, 2012, pp. 143-150). La duda que a veces suscitan es si, a fuerza de magnificar el espectáculo, acabarán distorsionando la imagen que del arte operístico se formarán los públicos potenciales. Lo que es seguro es que tales productos facilitan que sean los usuarios quienes seleccionen los contenidos de interés, los recomienden y, al enfatizar aquello que les ha provocado atracción, los tornen actuales. Por la manera personalizada que tienen de hacerlo, enseñan a sus pares a valorar un contenido que estaba alejado de su contexto familiar y actual. Es ese un proceso que está llamado a ser decisivo para atraer a nuevos públicos (Moreno-Cámara; Prieto-Gutiérrez, 2011).

4) La ópera intenta actualizarse como mundo de referencia también a través de las redes sociales. Además de alimentar y renovar la información sobre su universo, con ellas quiere evocar otra de las características esenciales de su arte: la experiencia en comunidad. En 2012 la mayoría de los teatros ya disponen de cuentas de Facebook y Twitter. Muchos de ellos actualizan a diario sus entradas. Sin embargo, en nuestro análisis hemos podido comprobar que en esos canales todavía no se crean auténticas comunidades virtuales alrededor de las producciones. Los teatros no acostumbran a responder a los mensajes que cuelgan los fans y el público en general. Tampoco suelen provocar el diálogo, ni organizar actividades para la comunidad o lanzar noticias y promociones exclusivas. Sólo los divos y divas del espectáculo parecen otorgar un uso más personal a estas plataformas (Radigales; Villanueva, 2011). Con esa participación de las estrellas, el star system adquiere en las redes sociales una nueva dimensión. Los artistas proyectan una imagen más humanizada de sí mismos y de la ópera ${ }^{6}$, lo cual provoca un visible interés compartido y contribuye a multiplicar el número de sus seguidores. Los organismos del arte operístico deberían aprender de ellos y dotar a las redes de una utilidad más propia. No hacerlo es desperdiciar una gran oportunidad de atraer, conocer, entender y adaptarse a sus nuevas audiencias (Moreno-Cámara; 
Prieto-Gutiérrez, 2011). Es dialogando con ellas como aprenderán a fidelizarlas y a provocar en ellas emociones compartidas (Gordillo, 2008, pp. 9-10). Al estimular un intercambio natural de conocimientos, inquietudes y deseos, las redes sociales les permitirían recrear emociones y suscitar auténticas implicaciones personales con su arte (Pereira-Domínguez; Urpí-Guercia, 2005, p. 78; Rössel, 2011, p. 89).

\section{Conclusión}

La ópera siente que su problema de actualidad es un problema de integración en la sociedad. Utiliza el vídeo e internet para sacar su espectáculo de los teatros e incorporarlo a la vida de nuevos públicos más mediáticos. Su nueva comunicación digital y audiovisual permite recrear en ellos la presencialidad y la viveza del espectáculo original. El diálogo que la ópera ha entablado con la tecnología marcará su futuro. Pero debe ponerse al servicio de un diálogo superior. A la ópera le queda el reto de emular en la Red su tercera nota esencial: la experiencia artística en comunidad. Si lo consigue, los nuevos contenidos operísticos -digitales y audiovisuales- que está generando, podrán ser compartidos y enriquecidos de manera más natural. De ser así, la ópera no sólo ampliaría sus públicos, sino que estaría siendo actualizada espontáneamente por ellos. Si quiere fidelizar a las nuevas audiencias, la clave para la ópera no es tanto actualizarse como ser actualizada.

\section{Notas}

1. Los datos presentados en este artículo han sido extraídos de las páginas oficiales de los principales organismos creadores de ópera. Teatros: Teatro Real (Madrid), Gran Teatre del Liceu (Barcelona), Opéra National de Paris (Paris), The Metropolitan Opera (Nueva York), Teatro alla Scala (Milán), Sydney Opera House (Sydney), Wiener Staatsoper (Viena), Deutsche Oper Berlin (Berlin), Royal Opera House (Londres), Chicago Opera Theater (Chicago), Teatro Colón (Buenos Aires), Bolshoi Teather (Moscú), Opéra de Lyon (Francia); The Nederladse Opera (Paises Bajos). Festivales: Bayreuther Festspiele (Bayreuth), Glyndenbourne Festival (Sussex), Arena di Verona Festival (Verona), Salzburger Festspiele (Salzburgo), Rossini Opera Festival (Pésaro). Terceros agentes: Medici TV, Arte TV, Classical TV, I2Cat (Cataluña), GARR (Roma), Terena (Países Bajos), Nesta (Londres).

2. Consultar los intermedios de las retransmisiones en HD que el Met de Nueva York produce para los cines: http://www.youtube.com/watch?v=zNc6BGFvy08

3. En 2008 aparecían en internet los primeros vídeos íntimos de los divos:

https://www.youtube.com/watch?v=hObQanDkXVg

https://www.youtube.com/watch?v=IJOCbEaJjlO\&feature $=r$ elated

\section{4. http://www.youtube.com/watch?v=fnAfc1_yAZo\&featu re $=$ relmfu}

5. Un lipdub o lip dub ("doblaje de labios") es un vídeo musical realizado por un grupo de personas que sincronizan sus labios, gestos y movimientos con una canción popular o cualquier otra fuente musical (http://es.wikipedia.org/wiki) Lipdub).
Un flashmob, traducido literalmente de inglés como «multitud instantánea» (flash: destello, ráfaga; mob: multitud), es una acción organizada en la que un gran grupo de personas se reúne de repente en un lugar público, realiza algo inusual y luego se dispersa rápidamente. Suelen convocarse a través de los medios telemáticos (móviles e internet) (http:// es.wikipedia.org/wiki/Flashmob). Ejemplos: http://www.youtube.com/watch?v=bsLivg6byjM http://www.youtube.com/watch?v=i4IUNO6oHIQ

6. http://www.facebook.com/pages/Juan-Diego-Florez/36 643928379

http://www.facebook.com/RobertoAlagna.Tenor

\section{Bibliografía}

Adorno, Theodor W. "Ópera burguesa". En: Adorno, Theodor W. Escritos musicales I-III. Madrid: Ediciones Akal, 2006, pp. 25-40.

Alier, Roger. ¿Qué es esto de la ópera?. Barcelona: Ediciones Robinbook, 2008. ISBN: 9788496924635

Bakhshi, Hasan; Pugh, Angela. An analysis of applications for the Digital R\&D Fund for Arts and Culture.

http://www.nesta.org.uk/library/documents/DigitalRnD Fundv5.pdf

Berte, Katrien; Hauttekeete, Laurence; Mechant, Peter; Nulens, Gert. "Broadband for culture, a culture for broadband?" Observatorio (OBS*), 2010, v. 4, n. 2, pp. 119-135.

Calvi, Juan; Santini, Rose-Marie. Música y audio/visual en internet. Hacia la configuración de nuevos hábitos de creación, difusión y consumo cultural en red.

http://rephip.unr.edu.ar/bitstream/handle/2133/1693/Tra bajo\%20Completo_\%20PN48.pdf?sequence $=1$

Carroll, Julie-Anne; Foth, Marcus; Adkins, Barbara. "Traversing urban social spaces: how online research helps unveil offline practice". En: Hunsinger, Jeremy; Klastrup, Lisbeth; Allen, Matthew. International handbook of internet research. New York: Springer Netherlands, 2010, pp. 147158. ISBN: 9781402097881

http://dx.doi.org/10.1007/978-1-4020-9789-8

Chavarría, Maricel. Opera en la era Twitter. http://hemerotecadigital.institutdelteatre.cat/jspui/bits tream/65324/26218/1/Article\%20diari.pdf

Colbert, François; Cuadrado, Manuel. Marketing de las artes y la cultura. Barcelona: Ariel, 2003. ISBN: 97884334 66993

De-Diego, Antonio. "Nuevas estrategias comunicativas en ópera por la inclusión del 'making of' en los DVDs". En: Intl workshop on opera \& video, 2010, pp. 1-14.

Eco, Umberto. Obra abierta. Barcelona: Planeta-De Agostini, 1985. ISBN: 8439500084

Fülfe, Ramona. Kulturmarketing: Impulse für eine zielgruppengerechte Ansprache im Bereich E-Musik. Hamburg: Diplomica Verlag, 2011. ISBN: 9783842854765

García-Avilés, José-Alberto. “La comunicación ante la con- 
vergencia digital". Signo y pensamiento, 2009, n. 54, pp. 102-113.

Gordillo, Inmaculada. "La hibridación en las nuevas formas dramáticas y espectaculares del siglo XXI". Icono14, 2008, n. 10, pp. 1-20.

Heyer, Paul. "Live from the Met: digital broadcast cinema, medium theory, and opera for the masses". Canadian journal of communication, 2008, n. 33, pp. 591-604.

Hoffmann, Bernward. "Medien und Biografie: 'Sie sind ein Stück von Deinem Leben'”. En: Hölzle, Christina; Jansen, Irma. Ressourcenorientierte Biografiearbeit. Grundlagen - Zielgruppen - Kreative Methoden. Wiesbaden: VS Verlag, 2011, pp. 273-278. ISBN: 9783531177748

http://dx.doi.org/10.1007/978-3-531-92623-0_18

Huang, Cheng; Li, Jin; Ross, Keith. "Can internet video on demand be profitable?". ACM Sigcomm computer communication review, 2007, v. 37, n. 4.

http://dx.doi.org/10.1145/1282427.1282396

Mclean, Fiona. "Services marketing: the case of museums". The service industries journal, 1994, v. 14, n. 2, pp. 190203.

http://dx.doi.org/10.1080/02642069400000022

Merten, Klaus. Einführung in die Kommunikationswissenschaft. Bd. 1: Grundlagen der Kommunikationswissenschaft. Berlin: Lit Verlag, 1999. ISBN: 9783894735920

Moreno-Cámara, Alicia; Prieto-Gutiérrez, Juan-José. “Instituciones culturales en un click". Razón y palabra, 2011, n. 78.

http://www.razonypalabra.org.mx/varia/N78/2a parte/25_ MorenoPrieto_V78.pdf

Muro, Robert. "Pecados y virtudes de la comunicación en las artes escénicas en España". En: Gómez-de-la-Iglesia, Roberto. La comunicación en la gestión cultural. Madrid: Grupo Xabide, 2006, pp. 155-164.

Orihuela, José-Luís. "Internet: nuevos paradigmas de la comunicación". Revista latinoamericana de comunicación Chasqui, 2002, n. 77.

http://redalyc.uaemex.mx/redalyc/pdf/160/16007702.pdf

Parker, Roger. Historia ilustrada de la ópera. Barcelona: Paidós Ibérica, 1998. ISBN: 9788449306334

Pereira-Domínguez, Carmen; Urpí-Guercia, Carmen. "Cine y juventud: una propuesta educativa integral". Revista de estudios de juventud, 2005, n. 68, pp. 73-89.
Radigales, Jaume. L'òpera. Música, teatre i espectacle. Barcelona: Enciclopedia Catalana, 1999. ISBN: 978 8473065368

Radigales, Jaume; Villanueva, Isabel. "Ópera y cine en España: la ópera como producto audiovisual. Retos para el siglo XXI". En: VI Simposio internacional de música y cine, 2011.

Rössel, Jörg. "Cultural capital and the variety of modes of cultural consumption in the opera audience". The sociological quarterly, 2011, n. 52, pp. 83-103.

http://dx.doi.org/10.1111/j.1533-8525.2010.01192.x

Roselli, John. "La ópera como acontecimiento social". En: Parker, Roger. Historia ilustrada de la ópera. Barcelona: Paidós Ibérica, 1998, pp. 450-482. ISBN: 9788449306334

Rhurmann, Georg. "Aktualität und Publizität revisited: Nachrichtenfaktoren und Beachtungsgrad von TV-Meldungen amb Beispiel des Themas 'Migranten'”. En: Wienand, Edith; Westerbarkey, Joachim; Scholl, Armin. Kommunikation über Kommunikation: Theorien, Methoden und Praxis. Festschrift für Klaus Merten. Wiesbaden: VS Verlag, 2005, pp. 67-82. ISBN: 9783531148717

Serra, Artur. "Cultural ring Latinoamerica-Europe, Tein3 and other live experiences". En: Network performing arts production workshop, 2011.

Scheff, Joanne; Kotler, Philip. "Crisis in the arts: the marketing response". California management review, 1996, v. 39, n. 1, pp. 28-52.

Sheil, Áine. "The opera director's voice: DVD 'Extras' and the question of authority". En: Pérez, J. Héctor. Opera and video. technology and spertatorship. Berna: Peter Lang, 2012, pp. 129-150. ISBN: 9783034305426.

Silverstone, Roger. Television and everyday life. New York: Routledge, 1994. ISBN: 0415016460

Smith, Janice. "Opera as an interdisciplinary art". Music educators journal, 1993, n. 79, pp. 21-61.

http://dx.doi.org/10.2307/3398544

Tambling, Jeremy. Opera, ideology and film. New York: St. Martin's Press, 1987.

Viacom. Tapping into tabletomics.

http://www.viacom.com/news/Pages/newstext.aspx?RID= 664831

Wasserman, Adam. Sight and sound: most valuable player. http://www.operanews.com/Opera_News_Magazine/ 2009/12/Departments/Sight_and_Sound_Most_Valua ble_Player.html

\section{Suscripción EPI sólo online}

Pensando sobre todo en los posibles suscriptores latinoamericanos, ya no es obligatorio pagar la suscripción impresa de EPI para acceder a la online.

EPI se ofrece a instituciones en suscripción "sólo online" a un precio considerablemente más reducido (95,19 + IVA euros/año), puesto que en esta modalidad no hay que cubrir los gastos de imprenta ni de correo postal. 